\title{
Sequential Algorithms for Max-Min Fair Bandwidth Allocation
}

\author{
Włodzimierz Ogryczak and Tomasz Śliwiński
}

\begin{abstract}
Telecommunication networks are facing the increasing demand for Internet services. Therefore, a problem of network dimensioning with elastic traffic arises which requires to allocate bandwidth to maximize service flows with fair treatment of all the services. In such applications, the so-called Max-Min Fairness (MMF) solution concept is widely used to formulate the resource allocation scheme. It assumes that the worst service performance is maximized and the solution is additionally regularized with the lexicographic maximization of the second worst performance, the third one etc. Due to lexicographic maximization of ordered quantities, the MMF solution concept cannot be tackled by the standard optimization model. It can be formulated a sequential lexicographic optimization procedure. Unfortunately, the basic sequential procedure is applicable only for convex models, thus it allows to deal with basic design problems but fails if practical discrete restrictions commonly arriving in telecommunications network design are to be taken into account. In this paper we analyze alternative sequential approaches allowing to solve non-convex MMF network dimensioning problems. Both the approaches are based on sequential optimization of directly defined artificial criteria. The criteria can be introduced into the original model with some auxiliary variables and linear inequalities thus the methods are easily implementable.
\end{abstract}

Key words: network design, resource allocation, fairness, lexicographic optimization, lexicographic max-min

Włodzimierz Ogryczak

Warsaw University of Technology, Institute of Control \& Computation Engineering, 00-665 Warsaw, Poland, e-mail: wogrycza@ia.pw.edu.pl

Tomasz Śliwiński

Warsaw University of Technology, Institute of Control \& Computation Engineering, 00-665 Warsaw, Poland, e-mail: tsliwins@ia.pw.edu.pl 


\section{Introduction}

A fair way of the bandwidth distribution among competing demands becomes a key issue in computer networks [3] and telecommunications network design, in general $[17,19,7,8]$. Due to increasing demand for Internet services, a problem of network dimensioning with elastic traffic arises which requires to allocate bandwidth to maximize service flows with fair treatment of all the services [17]. The problem of network dimensioning with elastic traffic can be formulated as follows [16]. Given a network topology $G=<V, E>$, consider a set of pairs of nodes as the set $J=\{1,2, \ldots, m\}$ of services representing the elastic flow from source $v_{j}^{s}$ to destination $v_{j}^{d}$. For each service, we have given the set $P_{j}$ of possible routing paths in the network from the source to the destination. This can be represented in the form of binary matrices $\Delta_{e}=\left(\delta_{e j p}\right)_{j \in J ; p \in P_{j}}$ assigned to each link $e \in E$, where $\delta_{e j p}=1$ if link $e$ belongs to the routing path $p \in P_{j}$ (connecting $v_{j}^{s}$ with $v_{j}^{d}$ ) and $\delta_{e j p}=0$ otherwise. For each service $j \in J$, the elastic flow from source $v_{j}^{s}$ to destination $v_{j}^{d}$ is a variable representing the model outcome and it will be denoted by $x_{j}$. This flow may be realized along various paths $p \in P_{j}$ and it is modeled as $x_{j}=\sum_{p \in P_{j}} x_{j p}$ where $x_{j p}$ are nonnegative variables representing the elastic flow from source $v_{j}^{s}$ to destination $v_{j}^{d}$ along the routing path $p \in P_{j}$. The single-path model requires additional multiple choice constraints to enforce nonbifurcated flows.

The network dimensioning problem depends on allocating the bandwidth to several links in order to maximize flows of all the services (demands). For each link $e \in E$ decision variables $\xi_{e} \geq 0$ represent the bandwidth allocated to link $e \in E$. Certainly, there are usually some bounds (upper limits) on possible expansion of the links capacities: $\xi_{e} \leq \bar{a}_{e}$ for all $e \in E$. Finally, the following constraints must be fulfilled:

$$
\begin{gathered}
0 \leq x_{j p} \leq M u_{j p} \quad \forall j \in J ; p \in P_{j} \\
u_{j p} \in\{0,1\} \quad \forall j \in J ; p \in P_{j} \\
\sum_{p \in P_{j}} u_{j p}=1 \quad \forall j \in J \\
\sum_{p \in P_{j}} x_{j p}=x_{j} \quad \forall j \in J \\
\sum_{j \in J} \sum_{p \in P_{j}} \delta_{e j p} x_{j p} \leq \xi_{e} \quad \forall e \in E \\
0 \leq \xi_{e} \leq \bar{a}_{e} \quad \forall e \in E \\
\sum_{e \in E} c_{e} \xi_{e} \leq B
\end{gathered}
$$

where (1a)-(1d) represent single-path flow requirements using additional binary (flow assignment) variables $u_{j p}$ and define the total service flows. Next, (1e) establish the relation between service flows and links bandwidth. The quantity $y_{e}=$ $\sum_{j \in J} \sum_{p \in P_{j}} \delta_{e j p} x_{j p}$ is the load of link $e$ and it cannot exceed the available link ca- 
pacity. Further, while allocating the bandwidth to several links the decisions must keep the cost within available budget $B(1 \mathrm{~g})$ where for each link $e \in E$ the cost of allocated bandwidth is $c_{e}$.

The network dimensioning model can be considered with various objective functions, depending on the chosen goal. Typically, the fairness requirement is formalized with the lexicographic maximinimization (lexicographic Max-Min approach). Within the telecommunications or network applications the lexicographic Max-Min approach has appeared already in [4] and now under the name Max-Min Fair (MMF) is treated as one of the standard fairness concepts $[2,6,10,17,20]$. Indeed, the MMF approach generalizes equal sharing at a single link bandwidth to any network allowing also to maximize the second smallest flows provided that the smallest remain optimal, the third smallest, etc.

The lexicographic maximinimization can be seen as searching for a vector lexicographically maximal in the space of the feasible vectors with components rearranged in the non-decreasing order. This can be mathematically formalized as follows. Let $\langle\mathbf{a}\rangle=\left(a_{\langle 1\rangle}, a_{\langle 2\rangle}, \ldots, a_{\langle m\rangle}\right)$ denote the vector obtained from $\mathbf{a}$ by rearranging its components in the non-decreasing order. That means $a_{\langle 1\rangle} \leq a_{\langle 2\rangle} \leq \ldots \leq a_{\langle m\rangle}$ and there exists a permutation $\pi$ of set $J$ such that $a_{\langle j\rangle}=a_{\pi(j)}$ for $j=1,2, \ldots, m$. Comparing lexicographically such ordered vectors $\langle y\rangle$ one gets the so-called lex-min order. The MMF problem can be then represented in the following way:

$$
\operatorname{lex} \max \left\{\left(y_{\langle 1\rangle}, y_{\langle 2\rangle}, \ldots, y_{\langle m\rangle}\right): y \in A\right\}
$$

where $A$ where depicts the set of attainable outcomes defined with constraints (1). Actually, we focus our analysis on the MMF bandwidth allocation problem but the approaches developed can be applied to various lexicographic Max-Min optimization problems, i.e., to problem (2) with various attainable sets $A$.

The (point-wise) ordering of outcomes causes that the lexicographic Max-Min problem (2) is, in general, hard to implement. Note that the quantity $y_{\langle 1\rangle}$ representing the worst outcome can be easily computed directly by the maximization:

$$
y_{\langle 1\rangle}=\max \left\{r_{1}: r_{1} \leq y_{j} \quad \forall j \in J\right\} .
$$

Similar simple formula does not exist for the further ordered outcomes $y_{\langle i\rangle}$. Nevertheless, for convex problems it is possible to build sequential algorithms for finding the consecutive values of the (unknown) MMF optimal outcome vector. While solving Max-Min problems for convex models there exists at least one blocked outcome which is constant on the entire set of optimal solutions to the Max-Min problem. Hence, the MMF solution can be found by solving a sequence of properly defined Max-Min problems with fixed outcomes (flows) that have been blocked by some critical constraints (link capacities) [12]. Indeed, in the case of LP models it leads to efficient algorithms taking advantages of the duality theory for simple identification of blocked outcomes $[1,5,18]$. Unfortunately, in our network dimensioning model it applies only to the basic LP constraints (1d)-(1g). In the case of nonconvex feasible set, such a blocked quantity may not exist [11] which makes the approach not applicable to our case of nonbifurcated flows enforced by discrete constraints (1a)- 
(1c). This can be illustrated with the simplified network depicted in Fig. 1 with link capacity limits given in the figure, cost coefficients 4 for links $\left(v_{1}, v_{5}\right), 3$ for $\left(v_{3}, v_{5}\right)$ and all other equal 1 , and the budget $\mathrm{B}=11$. We consider two demands: one connecting $v_{1}$ with $v_{2}$ along two possible paths $\left(v_{1}, v_{2}\right)$ or $\left(v_{1}, v_{5}, v_{6}, v_{2}\right)$; second connecting $v_{3}$ with $v_{4}$ along two possible paths $\left(v_{3}, v_{4}\right)$ or $\left(v_{3}, v_{5}, v_{6}, v_{4}\right)$. The MMF solution is unique and it allocates flow 1 to path $\left(v_{1}, v_{2}\right)$ (first demand) while flow 2 to path $\left(v_{3}, v_{5}, v_{6}, v_{4}\right)$ (second demand). The Max-Min (single-path) problem leads us to the conclusion that one of two flows cannot exceed 1 but not allowing us to identify which one must be blocked. Note that the same difficulty arrives also for the single path problem without any budget constraint, though the optimal solution is then not unique.

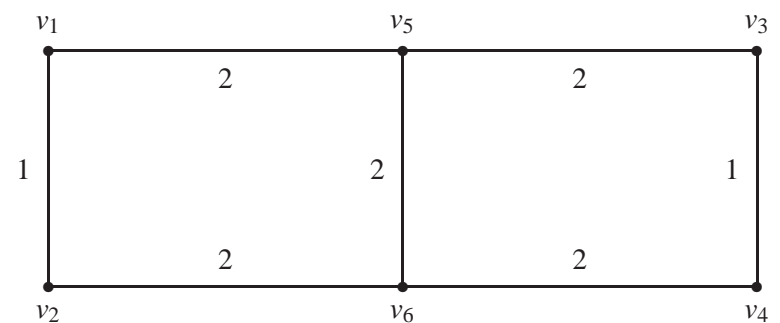

Fig. 1 Sample network without any critical link and blocked flow for Max-Min solution.

In this paper we analyze alternative sequential approaches allowing to solve nonconvex MMF network dimensioning problems. Both the approaches are based on the lexicographic optimization of directly defined artificial criteria. The criteria can be introduced into the original model with some auxiliary variables and linear inequalities independently from the problem structure.

\section{Cumulated Ordered Outcomes}

The point-wise ordering of outcomes for lexicographic optimization within the MMF problem (2) is, in general, hard to implement. Following Yager [21], a direct, although requiring the use of integer variables, formula can be given for any $y_{\langle i\rangle}$. Namely, for any $k=1,2, \ldots, m$ the following formula is valid:

$$
\begin{aligned}
y_{\langle i\rangle}= & \max r_{i} \\
& \text { s.t. } \\
& r_{i}-y_{j} \leq C z_{i j} \quad \forall j \in J \\
& z_{i j} \in\{0,1\} \quad \forall j \in J \\
& \sum_{j \in J} z_{i j} \leq i-1
\end{aligned}
$$

where $C$ is a sufficiently large constant (larger than any possible difference between various individual outcomes $y_{j}$ ) which allows us to enforce inequality $r_{i} \leq y_{j}$ for 
$z_{i j}=0$ while ignoring it for $z_{i j}=1$. Note that for $i=1$ all binary variables $z_{1 j}$ are forced to 0 thus reducing the optimization in this case to the standard LP model. However, for any other $i>1$ all $m$ binary variables $z_{i j}$ are an important part of the model. Nevertheless, with the use of auxiliary integer variables, any MMF problem (either convex or non-convex) can be formulated as the standard lexicographic maximization with directly defined objective functions

$$
\begin{aligned}
& \operatorname{lex} \max \left(r_{1}, r_{2}, \ldots, r_{m}\right) \\
& \text { s.t. } \\
& y \in A \\
& r_{i}-y_{j} \leq C z_{i j}, \quad \forall i, j \in J \\
& z_{i j} \in\{0,1\} \quad \forall i, j \in J \\
& \sum_{j \in J} z_{i j} \leq i-1 \quad \forall i \in J .
\end{aligned}
$$

We will refer to the above model as the Direct Ordered Outcomes (DOO) approach. Unfortunately, binary variables $z_{i j}$ in the auxiliary constraints contribute to implementation difficulties of the DOO approach.

There is, however, a way to reformulate the MMF problem (2) so that only linear variables are used. Let us consider cumulated criteria $\bar{\theta}_{i}(\mathbf{y})=\sum_{k=1}^{i} y_{\langle k\rangle}$ expressing, respectively: the worst (smallest) outcome, the total of the two worst outcomes, the total of the three worst outcomes, etc. Within the lexicographic optimization a cumulation of criteria does not affect the optimal solution. Hence, the MMF problem (2) can be formulated as the standard lexicographic maximization with cumulated ordered outcomes:

$$
\operatorname{lex} \max \left\{\left(\bar{\theta}_{1}(\mathbf{y}), \bar{\theta}_{2}(\mathbf{y}), \ldots, \bar{\theta}_{m}(\mathbf{y})\right): \mathbf{y} \in A\right\}
$$

Note that for any given vector $y \in \mathbb{R}^{m}$, the cumulated ordered value $\bar{\theta}_{i}(y)$ can be found as the optimal value of the following LP problem:

$$
\begin{aligned}
\bar{\theta}_{i}(y)= & \min \sum_{j \in J} y_{j} u_{i j} \\
& \text { s.t. } \\
& \sum_{j \in J} u_{i j}=k \\
& 0 \leq u_{i j} \leq 1 \quad \forall j \in J .
\end{aligned}
$$

The above problem is an LP for a given outcome vector $y$ while it becomes nonlinear for $y$ being a variable. This difficulty can be overcome by taking advantage of the LP duality. Note that the LP dual of problem (5) with variable $r_{i}$ corresponding to the equation $\sum_{j \in J} u_{i j}=k$ and variables $d_{i j}$ corresponding to upper bounds on $u_{i j}$ leads us to the following formula:

$$
\begin{aligned}
& \bar{\theta}_{i}(y)=\max i r_{i}-\sum_{j \in J} d_{i j} \\
& \text { s.t. } \\
& r_{i}-y_{j} \leq d_{i j}, d_{i j} \geq 0 \quad \forall j \in J .
\end{aligned}
$$


It follows from (6) that $\bar{\theta}_{k}(y)=\max \left\{k r_{k}-\sum_{j \in J}\left(y_{j}-r_{k}\right)_{+}: y \in A\right\}$ where $(.)_{+}$ denotes the nonnegative part of a number and $r_{k}$ is an auxiliary (unbounded) variable. The latter, with the necessary adaptation to the minimized outcomes in location problems, is equivalent to the computational formulation of the $k$-centrum model introduced in [15]. Hence, the LP dual transformation provides an alternative proof of that formulation.

Following (6), we may express the MMF problem (2) as a standard lexicographic optimization problem with predefined linear criteria:

$$
\begin{aligned}
& \operatorname{lex} \max \left(r_{1}-\sum_{j \in J} d_{1 j}, 2 r_{2}-\sum_{j \in J} d_{2 j}, \ldots, m r_{m}-\sum_{j \in J} d_{m j}\right) \\
& \text { s.t. } \\
& y \in A \\
& d_{i j} \geq r_{i}-y_{j} \quad \forall i, j \in J \\
& d_{i j} \geq 0 \quad \forall i, j \in J .
\end{aligned}
$$

We will refer to the above model as the Cumulated Ordered Outcomes (COO) approach.

Theorem 1. An attainable outcome vector $y \in A$ is an optimal solution of the MMF problem (2), if and only if it is an optimal solution of the COO model (7).

Note that this direct lexicographic formulation of the COO model remains valid for nonconvex (e.g. discrete) models, where the standard sequential approaches [9] are not applicable. Model COO preserves the problem convexity when the original problem is defined with convex feasible set $A$. In particular, for an LP original problem it remains within the LP class while introducing $m^{2}+m$ auxiliary variables and $m^{2}$ constraints. Thus, for many problems with not too large number of services (demands) $m$, problem (7) can easily be solved directly.

\section{Shortfalls to Ordered Targets}

For some specific classes of discrete, or rather combinatorial, optimization problems, one may take advantage of the finiteness of the set of all possible outcome values. The ordered outcome vectors may be treated as describing a distribution of outcomes $y$. In the case when there exists a finite set of all possible outcomes, we can directly describe the distribution of outcomes with frequencies of outcomes. Let $V=\left\{v_{1}, v_{2}, \ldots, v_{r}\right\}$ (where $v_{1}<v_{2}<\cdots<v_{r}$ ) denote the set of all attainable outcomes. We introduce integer functions $h_{k}(y)(k=1, \ldots, r)$ expressing the number of values $v_{k}$ in the outcome vector $y$. Having defined functions $h_{k}$ we can introduce cumulative distribution functions:

$$
\bar{h}_{k}(y)=\sum_{l=1}^{k} h_{l}(y), \quad k=1, \ldots, r .
$$


Function $\bar{h}_{k}$ expresses the number of outcomes smaller or equal to $v_{k}$. Since we want to maximize all the outcomes, we are interested in the minimization of all functions $\bar{h}_{k}$. Indeed, the following assertion is valid [11]. For outcome vectors $y^{\prime}, y^{\prime \prime} \in V^{m}$, $\left\langle y^{\prime}\right\rangle \geq\left\langle y^{\prime \prime}\right\rangle$ if and only if $\bar{h}_{k}\left(y^{\prime}\right) \leq \bar{h}_{k}\left(y^{\prime \prime}\right)$ for all $k=1, \ldots, r$. This equivalence allows to express the MMF problem (2) in terms of the standard lexicographic minimization problem with objectives $\overline{\mathbf{h}}(y)$ :

$$
\operatorname{lex} \min \left\{\left(\bar{h}_{1}(y), \ldots, \bar{h}_{r}(y)\right): y \in A\right\} .
$$

Theorem 2. An attainable outcome vector $y \in A$ is an optimal solution of the MMF problem (2), if and only if it is an optimal solution of the lexicographic problem (9).

The quantity $\bar{h}_{k}(y)$ can be computed directly by the minimization:

$$
\begin{aligned}
& \bar{h}_{k}(y)=\min \sum_{j \in J} z_{k j} \\
& \text { s.t. } v_{k+1}-y_{j} \leq C z_{k j}, z_{k j} \in\{0,1\} \quad \forall j \in J,
\end{aligned}
$$

where $C$ is a sufficiently large constant. Note that $\bar{h}_{r}(y)=m$ for any $y$ which means that the $r$-th criterion is always constant and therefore redundant in (9). Hence, the lexicographic problem (9) can be formulated as the following mixed integer problem:

$$
\begin{aligned}
& \operatorname{lex} \min \left(\sum_{j \in J} z_{1 j}, \sum_{j \in J} z_{2 j}, \ldots, \sum_{j \in J} z_{r-1, j}\right) \\
& \text { s.t. } \\
& v_{k+1}-y_{j} \leq C z_{k j} \quad j \in J, k<r \\
& z_{k j} \in\{0,1\} \quad j \in J, k<r \\
& y \in A .
\end{aligned}
$$

Taking advantage of possible weighting and cumulating achievements in lexicographic optimization, one may eliminate auxiliary integer variables from the achievement functions. For this purpose we weight and cumulate vector $\overline{\mathbf{h}}(y)$ to get $\hat{h}_{1}(y)=0$ and:

$$
\hat{h}_{k}(y)=\sum_{l=1}^{k-1}\left(v_{l+1}-v_{l}\right) \bar{h}_{l}(y) \quad k=2, \ldots, r .
$$

Due to positive differences $v_{l+1}-v_{l}>0$, the lexicographic minimization problem (9) is equivalent to the lexicographic problem with objectives $\hat{\mathbf{h}}(y)$ :

$$
\operatorname{lex} \min \left\{\left(\hat{h}_{1}(y), \ldots, \hat{h}_{r}(y)\right): y \in A\right\}
$$

which leads us to the following assertion.

Theorem 3. An attainable outcome vector $y \in A$ is an optimal solution of the MMF problem (2), if and only if it is an optimal solution of the lexicographic problem (12). 
Actually, vector function $\hat{\mathbf{h}}(y)$ provides a unique description of the distribution of coefficients of vector $y$, i.e., for any $y^{\prime}, y^{\prime \prime} \in V^{m}$ one gets: $\hat{\mathbf{h}}\left(y^{\prime}\right)=\hat{\mathbf{h}}\left(y^{\prime \prime}\right) \Leftrightarrow$ $\left\langle y^{\prime}\right\rangle=\left\langle y^{\prime \prime}\right\rangle$. Moreover, $\hat{\mathbf{h}}\left(y^{\prime}\right) \leq \hat{\mathbf{h}}\left(y^{\prime \prime}\right)$ if and only if $\bar{\Theta}\left(y^{\prime}\right) \geq \bar{\Theta}\left(y^{\prime \prime}\right)[11]$.

Note that $\hat{h}_{1}(y)=0$ for any $y$ which means that the first criterion is constant and redundant in problem (12). Moreover, putting (8) into (11) allows us to express all achievement functions $\hat{h}_{k}(y)$ as a piecewise linear functions of $y$ :

$$
\hat{h}_{k}(y)=\sum_{j \in J} \max \left\{v_{k}-y_{j}, 0\right\} \quad k=1, \ldots, r
$$

Hence, the quantity $\hat{h}_{k}(y)$ can be computed directly by the following minimization:

$$
\begin{aligned}
\hat{h}_{k}(y) & =\min \sum_{j \in J} t_{k j} \\
& \text { s.t. } \\
& v_{k}-y_{j} \leq t_{k j}, t_{k j} \geq 0 \quad \forall j \in J .
\end{aligned}
$$

Therefore, the entire lexicographic model (12) can be formulated as follows:

$$
\begin{aligned}
& \operatorname{lex} \min \left(\sum_{j \in J} t_{2 j}, \sum_{j \in J} t_{3 j}, \ldots, \sum_{j \in J} t_{r j}\right) \\
& \text { s.t. } \\
& v_{k}-y_{j} \leq t_{k j} \quad j \in J, k=2, \ldots, r \\
& t_{k j} \geq 0 \quad j \in J, \quad k=2, \ldots, r \\
& y \in A .
\end{aligned}
$$

We will refer to the above model as the Shortfalls to Ordered Targets (SOT) approach.

Note that the above formulation, unlike the problem (10), does not use integer variables and can be considered as an LP modification of the original constraints (1). Thus, this model preserves the problem's convexity when the original problem is defined with a convex set $A$. The size of problem (15) depends on the number of different outcome values. Thus, for many problems with not too large number of outcome values, the problem can easily be solved directly. Note that in many problems of telecommunications network design, the objective functions express the quality of service and one can easily consider a limited finite scale (grid) of the corresponding outcome values. One may also notice that model (15) opens a way for the fuzzy representation of quality measures within the MMF problems.

\section{Computational experiments}

We have performed some initial tests of the sequential approaches to the MMF the network dimensioning problem (1). We have not assumed any bandwidth granulation and thereby the grid of possible bandwidth values that can be allocated. There- 
fore, in case of the Shortfalls to Ordered Targets approach the resulting bandwidth allocation is only an approximation to the exact MMF solution.

For the experiments we used a set of 10 randomly generated problems for each tested size. The problems were generated as follows. First, we created random but consistent network structure. Then, we chose random node pairs to define services. For each service 3 different possible flow routes between the two end nodes were generated. Two of them were fully random and one was the shortest path between the nodes (with smallest number of links). We decided to use the integer grid of the $v_{k}$ values in the ordered values approach, that is to check each integer value from the feasible set of objective values. In this case the number of targets depends on the range of the feasible objective values. We managed to restrict the number of targets to the range of 5 to 10 applying different link capacities for different problem sizes. We set the large budget limit $B$ thus relaxing the budget constraints (1g).

We analyzed the performance of the three sequential approaches: the Direct Ordered Outcomes (DOO) model (4), the Cumulated Ordered Outcomes (COO) model (7) and the Shortfalls to Ordered Targets (SOT) model (15) with the condition $y \in A$ representing the bandwidth allocation problem defined with constraints (1). Each model was computed using the standard sequential algorithm for lexicographic optimization with predefined objective functions. For lexicographic maximization problem lex $\max \left\{\left(g_{1}(y), \ldots, g_{m}(y)\right): y \in Y\right\}$ the algorithm reads as follows:

Step 0: Put $k:=1$.

Step 1: Solve problem $P_{k}$ :

$\max _{y \in Y}\left\{\tau_{k}: \tau_{k} \leq g_{k}(y), \tau_{j}^{0} \leq g_{j}(y) \forall_{j<k}\right\}$

denote the optimal solution by $\left(y^{0}, \tau_{k}^{0}\right)$.

Step 2: If $k=m, \mathbf{S T O P}\left(y^{0}\right.$ is MMF optimal).

Otherwise, put $k:=k+1$ and

go to Step 1.

For example, the algorithm for the COO model worked according to the above scheme with functions $g_{k}$ defined as $k t_{k}-\sum_{j \in J} d_{k j}$. Let $k=1$. Following (7), we built initial problem $P_{1}$ with the objective $\tau_{1}=t_{1}-\sum_{j \in J} d_{1 j}$ being maximized and $m$ constraints of the form $t_{1}-d_{1 j} \leq y_{j}, j=1 \ldots m$. The expression $y \in A$ of (7) was replaced by (1). Each new problem $P_{k}$ in subsequent iterations $(k>1)$ was built by adding new constraints $\tau_{k-1}^{0} \leq t_{k-1}-\sum_{j \in J} d_{k-1, j}$ and $t_{k}-d_{k j} \leq y_{j}, j=1 \ldots m$ to problem $P_{k-1}$, where $\tau_{k-1}^{0}$ was the optimal objective value of $P_{k-1}$. Similar algorithm was performed for the DOO as well as for the SOT approach. The difference was in the objectives and auxiliary constraints, as defined in (4) and (15), respectively. All the tests were performed on the Pentium IV $1.7 \mathrm{GHz}$ computer employing the CPLEX 9.1 package.

Table 1 presents solution times for the three approaches being analyzed. The times are averages of 10 randomly generated problems. The upper index denotes the number of tests out of 10 for which the timeout of 120 seconds occurred. The minus sign '-' shows that the timeout occurred for all 10 test problems. One can notice that while for smaller problems with number of services equal 5 all three approaches perform very well, for bigger problems only the SOT approach gives 
Table 1 Computation times (in seconds) for different solution approaches.

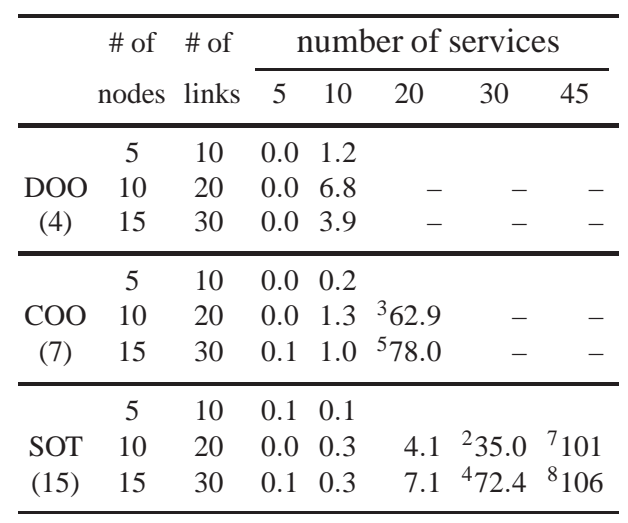

acceptable results (in the sense of solving majority of problems within 120 seconds time limit).

Table 2 Computation times (in seconds) for problems with increased link capacities.

\begin{tabular}{cccccccc}
\hline & \# of & \# of & \multicolumn{6}{c}{ number of services } \\
\cline { 4 - 9 } & & & 5 & 10 & 20 & 30 & 45 \\
\hline & 5 & 10 & 0.1 & 0.1 & & & \\
SOT & 10 & 20 & 0.1 & 1.3 & 23.8 & ${ }^{4} 74.3$ & - \\
$(15)$ & 15 & 30 & 0.1 & 1.2 & 33.9 & ${ }^{8} 108.0$ & - \\
\hline
\end{tabular}

To examine how the number of targets in the SOT approach influences the test results we also performed similar experiments increasing the capacities of links and considering 15 to 25 targets. This did not affected significantly the DOO and COO approaches. For the SOT approach the computing times increased (Tab. 2) but it still outperforms both DOO and COO. approaches.

\section{Conclusion}

As lexicographic maximization in the Max-Min Fair optimization is not applied to any specific order of the original outcomes, the MMF optimization can be very hard to implement in general nonconvex (possibly discrete) problems. We have shown that introduction of some artificial criteria with auxiliary variables and linear inequalities allows one to model and to solve the MMF problems in very efficient way. We have performed initial tests of computational performance of the presented 
models for the MMF network dimensioning problem. It turns out that both the models outperform the Direct Ordered Outcomes model. The Shortfall to Ordered Targets model enables to solve within 2 minutes majority of the MMF single path dimensioning problems for networks with 15 nodes and 30 links. Such performance is enough for efficient analysis of a country backbone network of ISP (12 nodes and 18 links in the case of Poland [14]). Nevertheless, further research is necessary on the models and corresponding algorithms tailored to specific MMF network optimization problems. The models may also be applied to various MMF resource allocation problems, not necessarily related to networks.

Acknowledgements The research was supported by the Ministry of Science and Information Society Technologies under grant 3T11C 005 27. "Models and Algorithms for Efficient and Fair Resource Allocation in Complex Systems".

\section{References}

1. F.A. Behringer, A simplex based algorithm for the lexicographically extended linear maxmin problem, Europ. J. Opnl, Res. 7, 1981, pp. 274-283.

2. D. Bertsekas and R. Gallager, Data Networks, Prentice-Hall, Englewood Cliffs 1987.

3. R. Denda, A. Banchs and W. Effelsberg, The fairness challenge in computer networks. LNCS 1922, 2000, pp. 208-220.

4. J. Jaffe, Bottleneck flow control, IEEE Trans. on Comm. 7, 1980, pp. 207-237.

5. R.S. Klein, H, Luss and U.G. Rothblum, Minimax resource allocation problems with resourcesubstitutions represented by graphs. Opns. Res. 41, 1993, pp. 959-971.

6. J. Kleinberg, Y. Rabani and E. Tardos, Fairness in routing and load balancing, J. Comput. Syst. Sci. 63, 2001, pp. 2-21.

7. C.Y. Lee and H.K. Choo, Discrete bandwidth allocation considering fairness and transmission load in multicast networks, Comp. \& Opns, Res, 34, 2007, pp. 884-899.

8. Ch. Linardakis, H.C. Leligou and J.D. Angelopoulos, Performance evaluation of a distributed credit-based fairness mechanism for slotted WDM rings, 8th WSEAS Int. Conf. on Communications 2004, paper 487-455.

9. H. Luss, On equitable resource allocation problems: a lexicographic minimax approach, Opns. Res. 47, 1999, pp. 361-378.

10. D. Nace, L.N. Doan, O. Klopfenstein and A. Bashllari, Max-min fairness in multi-commodity flows, Comp. \& Opns. Res. 35, 2008, pp. 557-573.

11. W. Ogryczak, Linear and Discrete Optimization with Multiple Criteria: Preference Models and Applications to Decision Support (in Polish), Warsaw Univ. Press, 1997.

12. W. Ogryczak, M. Pióro and A. Tomaszewski, Telecommunication network design and maxmin optimization problem. J. Telecomm. and Info. Tech. 3, 2005, pp. 43-56.

13. W. Ogryczak and T. Śliwiński. On Direct Methods for Lexicographic Min-Max Optimization, LNCS 3982, 2006, 802-811.

14. W. Ogryczak, T. Śliwiński and A. Wierzbicki, Fair Resource Allocation Schemes and Network Dimensioning Problems, J. Telecomm. and Info. Tech. 3, 2003, pp. 34-42.

15. W. Ogryczak and A. Tamir, Minimizing the sum of the $k$ largest functions in linear time, Info. Proc. Let. 85, 2003, pp. 117-122.

16. W. Ogryczak, A. Wierzbicki and M. Milewski, A multi-criteria approach to fair and efficient bandwidth allocation, Omega 36, 2008, pp. 451-463.

17. M. Pióro and D. Medhi, Routing, Flow and Capacity Design in Communication and Computer Networks. Morgan Kaufmann, San Francisco 2004. 
18. M. Pióro, P. Nilsson, E. Kubilinskas and G. Fodor, On efficient max-min fair routing algorithms, Proc. 8th IEEE ISCC'03, 2003, pp. 365-372.

19. R.M. Salles and J.A Barria, Fair and efficient dynamic bandwidth allocation for multiapplication networks, Comp. Networks 49, 2005, pp. 856-877.

20. R. de Silva, A simple approach to congestion control of ABR traffic with weighted Max-Min Fairness, WSEAS Trans. on Computers 3, 2004, pp. 75-78.

21. R.R. Yager, On the analytic representation of the Leximin ordering and its application to flexible constraint propagation, Europ. J. Opnl, Res. 102, 1997, pp. 176-192. 